\title{
Research shortcomings of fluorescent nanothermometers in biological and medical fields
}

\author{
Yang Yuexuan ${ }^{1} \&$ Wu Daocheng*,1 \\ ${ }^{1}$ Key Laboratory of Biomedical Information Engineering of Education Ministry, School of Life Science \& Technology, Xi'an Jiaotong \\ University, Xi'an, 710049, PR China \\ *Author for correspondence: Tel.: +86 298266 3941; Fax: +86 298266 3941; wudaocheng@mail.xjtu.edu.cn
}

\begin{abstract}
"Although fluorescent nanothermometers are effective and promising temperature measurement tools for bio-systems, there are still many difficulties remained and their theoretical research as well as actual application must be improved."
\end{abstract}

First draft submitted: 10 November 2019; Accepted for publication: 14 January 2020; Published online: 13 March 2020

Keywords: bio-systems $\bullet$ fluorescent nanothermometers $\bullet$ medicine $\bullet$ research shortcomings $\bullet$ temperature

Temperature is an important physical quantity that expresses the hotness or coldness degree of an object. It is an important index in biological and medical fields. The accurate measurement of temperature in the microareas of bio-systems like tissues, cells and organelles, such as mitochondria, can give an indication as to the biological conditions of such regions and also provides insights into their biochemical reactions and disease states. Luminescence (fluorescence, chemiluminescence, photoluminescence of plasmonic metal nanoparticles [1]) nanothermometers are nanosized temperature sensors with temperature-dependent luminescence properties. Such devices can be used to detect the temperature of micro-areas in biological systems.

Owing to their high sensitivity, spatial-temporal resolution and ease of visualization, fluorescent nanothermometers have attracted increasing attention among researchers in the fields of biology and medicine. In general, fluorescent nanothermometers use the temperature-sensitive characteristics of nanoparticle fluorescence, such as the changes in fluorescence intensity, emission peak position and fluorescence lifetime with the change in temperature to measure temperature [2]. At present, many types of fluorescent nanothermometers are available, which are based on organic fluorescent dyes, quantum dots, upconversion nanoparticles, luminescent lanthanide complexes, fluorescent proteins and diamonds [3-5]. These tools are useful for the research of life activities and medical phenomena at the submicroscopic scale. However, along with this deepening research, some shortcomings of fluorescent nanothermometers have recently appeared. As time progresses and the biological systems being measured are getting smaller and smaller (tissue-cells-organelles), there are many contradictions in space and time for accurate temperature measurement, which have caused a lot of discussions [6].

\section{Shortcomings of research on fluorescent nanothermometers}

The main shortcomings of research on fluorescent nanothermometers include the following:

\section{Improvement of detection sensitivity}

Sensitivity is an important parameter in the performance of fluorescent nanothermometers. A high sensitivity indicates accurate temperature measurement and a minimal change in the related biological characteristic detection. To date, the sensitivity of the current fluorescent nanothermometers is insufficient. For instance, the near-infrared emission $\mathrm{PbS} / \mathrm{CdS} / \mathrm{ZnS}$ quantum dots prepared by del Rosal et al. have reached a sensitivity of $1 \%{ }^{\circ} \mathrm{C}^{-1}[7]$. Xu et al. reported that the ratiometric nanothermometer based on triplet sensitized upconversion has a sensitivity of $7.1 \%{ }^{\circ} \mathrm{C}^{-1}[8]$. In our previous study, we prepared a fluorescent nanothermometer based on $\mathrm{CuInS}_{2} / \mathrm{ZnS}$ quantum dots with a sensitivity of $2 \%{ }^{\circ} \mathrm{C}^{-1}$ and temperature resolution is as high as $\pm 0.5^{\circ} \mathrm{C}$ [9]. Given the difficulty of developing fluorescent nanothermometers with a new temperature-sensitive mechanism, their sensitivity failed

Future Medicine 
to achieve remarkable improvement (most studies reported sensitivities of less than $10 \%{ }^{\circ} \mathrm{C}^{-1}$ ); hence, these nanothermometers cannot meet the needs for enhanced temperature detection in biological and medical fields. Detection sensitivity is also related to response time and detection range. In addition, the current fluorescent nanothermometers show poor reproducibility (affected by photobleaching, brightness, etc.), which considerably limits their applications in the fields of biology and medicine.

\section{Difficulty in measuring the real temperature}

Given the complexity of biological systems, a standard method for measuring the real temperature of the micro-areas has not been developed. Therefore, when applied in organism structures, such as cells or organelles, fluorescent nanothermometers cannot calibrate its real temperature measurement. The measurement results can only be represented by the temperature change $(\Delta \mathrm{T})$, especially in the micro-areas of medicine [10]. For example - in measuring the temperature of local tumor tissues, the current detection method, which is infrared imager temperature sensing, can only measure the skin temperature. The temperature within tumor tissues cannot be measured accurately and the real temperature of the tumor tissue cannot be compared with that obtained using other methods when the fluorescent nanothermometers are injected into the tumor tissue [11] or subcutaneously administered [12]. Thus, the measured temperature cannot accurately reflect the biological features of local tumor tissues.

\section{Limitations in biological and medicine applications}

The use of fluorescent nanothermometers in the fields of biology and medicine feature great limitations. Accurately measuring the temperature of the micro-areas of experimental animals using the current fluorescent nanothermometers presents difficulty. Among the existing applications, the measurement of cellular temperature change is easy to achieve $[13,14]$ and related reports have been found. However, the temperatures of other organelles, such as mitochondria, are difficult to measure. Given that the micro-area temperature represents a large number of biochemical reactions, measuring the temperature of each compartment separated by the double membrane of the mitochondria is also difficult. Chretien $e t$ al. reported that the temperature in mitochondria is approximately $10^{\circ} \mathrm{C}$ higher than that of subcellular organs in cells [15]. However, the reliability of this measurement remains low due to the lack of control. At present, most of the thermometry research focuses on organelles, cells and tissue, while limited studies have been conducted on the direct application of fluorescent nanothermometers in the measurement of animal temperature and none in clinical. Most of the common experimental animals (mice, rabbit etc.) are isothermal animals. Under the stimulation of an external environment, they can still maintain the temperature stability of the internal environment after a short period. In in vivo research, local laser irradiation [16] or microwave radiation [9] heating has been used to determine the temperature change in the part to be measured. However, the temperature can only be maintained within a short period because only the epidermis was heated, causing negligible effect on the temperature of the animals' body. Effectively changing the local temperature of experimental animals remains a problem to be solved. Fluorescent nanothermometers must be improved for application in a wide range of biology and medicine. In addition, much attention should be paid to toxicity of fluorescent nanothermometers, especially those containing heavy metals [17].

\section{Lack of theoretical research \& supporting instruments}

Tracing back to their origin, the studies on fluorescent nanothermometers encountered a bottleneck that was largely due to the lack of theoretical research. Thus, the measured parameters and the mechanism of temperature change both remain unclear. Given that the theory behind fluorescent nanothermometers involves life science, material science, chemistry, optics and quantum mechanics, the mechanism of temperature response is highly complex and extremely difficult to study. If a breakthrough is made in theoretical research, the studies on fluorescent nanothermometers may achieve considerable progress under the guidance of theories. Although fluorescence detection exhibits a high spatial-temporal resolution, the response of current detection instruments lacks speed due to the insufficient supporting instruments, which also affect the time resolution of fluorescence nanothermometers, resulting in their incapability to accurately obtain temperature response at the time of stimulation. Furthermore, data processing is relatively complex and is not conducive for the use of fluorescent nanothermometers. Given the lack of practical applications of such nanothermometer, the research on supporting instruments has not attracted attention. 


\section{Future perspective}

In view of these shortcomings of fluorescent nanothermometers, we realize that the following development trends will occur in the future. First, the technology of fluorescent nanothermometers is not developed perfectly, but shows good application prospect in biological and medicine fields. With the development of theoretical research, novel temperature change mechanism systems must be developed to improve the sensitivity and adaptability of fluorescent nanothermometers. A relative standard method that can accurately measure the local temperature might be developed. This method will provide reference for measurement using fluorescence nanothermometers and achieve real temperature values, providing convenience for the research on such nanothermometers. With regard to the current situation, theoretical research must be strengthened. Through molecular simulation studies, we can explore the temperature-sensitive mechanism of fluorescent nanothermometers and the specific relationship between optical parameters and temperature, forming a complete theoretical system for fluorescent nanothermometers that can be used as the cornerstone and reference of related research which can promote the studies on such nanothermometers. In addition, some new materials like nanoparticles whose emission is in NIR-II, NIR-III will be used in the biological and medical fields. Second, the existing application systems must be extended. Fluorescent nanothermometers can be used not only in living cells and bodies but also in solution systems to explore the characteristics of DNA, proteins, other biological macromolecules and the thermodynamics of various biochemical reactions. Application research in the medical field (diagnosis of pathological changes, monitoring of hyperthermia of tumors, etc.) can also be pushed further in-depth and clinical. Finally, special instruments and equipment must be developed to improve the parameter measurement of the supporting equipment for fluorescent nanothermometers to improve their sensitivity, actual detection limit, time resolution and other properties and take full advantage of the high sensitivity and high spatial-temporal resolution of fluorescent detection.

\section{Conclusion}

Overall, fluorescent nanothermometers are effective temperature measurement tools for bio-systems. Although fluorescent nanothermometers are effective and promising temperature measurement tools for bio-systems, there are still many difficulties remained and their theoretical research as well as actual application must be improved.

\section{Financial \& competing interests disclosure}

This work was supported by the National Natural Science Foundation of China (nos: 81471771 and 81871476 ). The authors have no other relevant affiliations or financial involvement with any organization or entity with a financial interest in or financial conflict with the subject matter or materials discussed in the manuscript apart from those disclosed.

No writing assistance was utilized in the production of this manuscript.

\section{References}

1. Carattino A, Caldarola M, Orrit M. Gold nanoparticles as absolute nanothermometers. Nano Lett. 18(2), 874-880 (2018).

2. Homma M, Takei Y, Murata A, Inoue T, Takeoka S. A ratiometric fluorescent molecular probe for visualization of mitochondrial temperature in living cells. Chem. Commun. 51(28), 6194-6197 (2015).

3. Kucsko G, Maurer P, Yao N et al. Nanometre-scale thermometry in a living cell. Nature 500(7460), 54-58 (2013).

4. Wu Y, Liu J, Ma J, Liu J, Wang Y, Wu D. Ratiometric nanothermometer based on rhodamine dye-incorporated F127-melamine-formaldehyde polymer nanoparticle: preparation, characterization, wide-range temperature sensing and precise intracellular thermometry. ACS Appl. Mater. Interfaces 8(23), 14396-14405 (2016).

5. Wu Y, Liu J, Wang Y et al. Novel ratiometric fluorescent nanothermometers based on fluorophores-labeled short single-stranded DNA. ACS Appl. Mater. Interfaces 9(12), 11073-11081 (2017).

6. Baffou G, Rigneault H, Marguet D, Jullien L. A critique of methods for temperature imaging in single cells. Nat. Methods 11(9), 899-901 (2014).

7. del Rosal B, Carrasco E, Ren F et al. Infrared-emitting QDs for thermal therapy with real-time subcutaneous temperature feedback. Adv. Funct. Mater. 26(33), 6060-6068 (2016).

8. $\mathrm{Xu} \mathrm{M,} \mathrm{Zou} \mathrm{X,} \mathrm{Su} \mathrm{Q} \mathrm{et} \mathrm{al.} \mathrm{Ratiometric} \mathrm{nanothermometer} \mathrm{in} \mathrm{vivo} \mathrm{based} \mathrm{on} \mathrm{triplet} \mathrm{sensitized} \mathrm{upconversion.} \mathrm{Nat.}$ Commun. 9(1), 2698-2714 (2018).

9. Zhang H, Wu Y, Gan Z et al. Accurate intracellular and in vivo temperature sensing based on CuInS $2 / \mathrm{ZnS}_{\mathrm{QD}}$ micelles. J. Mater. Chem. $B 7(17), 2835-2844$ (2019).

10. Takei Y, Arai S, Murata A et al. A nanoparticle-based ratiometric and self-calibrated fluorescent thermometer for single living cells. ACS Nano 8(1), 198-206 (2014). 
11. Carrasco E, del Rosal B, Sanzrodriguez F et al. Intratumoral thermal reading during photo-thermal therapy by multifunctional fluorescent nanoparticles. Adv. Funct. Mater. 25(4), 615-626 (2015).

12. Ximendes EC, Santos WQ, Rocha U et al. Unveiling in vivo subcutaneous thermal dynamics by infrared luminescent nanothermometers. Nano Lett. 16(3), 1695-1703 (2016).

13. Wang C, Ling L, Yao Y, Song Q. One step synthesis of fluorescent smart thermo-responsive copper clusters: a potential nanothermometer in living cells. Nano Res. 8(6), 1975-1986 (2015).

14. Wang C, Jiang K, Wu Q, Wu J, Zhang C. Green synthesis of red-emitting carbon nanodots as a novel "turn-on" nanothermometer in living cells. Chem. Eur. J. 22(41), 14475-14479 (2016).

15. Chretien D, Benit P, Ha H et al. Mitochondria are physiologically maintained at close to $50{ }^{\circ}$ C. PLoS Biol. 16(1), e2003992 (2018).

16. Ximendes EC, Rocha U, Sales TO et al. In vivo subcutaneous thermal video recording by supersensitive infrared nanothermometers. Adv. Funct. Mater. 27(38), 1702249 (2017).

17. Liu J, Zhang H, Selopal GS, Sun S, Zhao H, Rosei F. Visible and near-infrared, multiparametric, ultrasensitive nanothermometer based on dual-emission colloidal quantum dots. ACS Photonics 6(10), 2479-2486 (2019). 